\title{
EPIGENETIC SIGNATURE OF CHRONIC MATERNAL STRESS LOAD DURING PREGNANCY MIGHT BE A POTENTIAL BIOMARKER FOR SPONTANEOUS PRETERM BIRTH
}

\author{
Rogac $\mathrm{M}^{*}$, Peterlin B \\ *Corresponding Author: Mihael Rogac, M.D., Ph.D., Clinical Institute of Medical Genetics, Univer- \\ sity Medical Center Ljubljana, Slajmerjeva 4, 1000 Ljubljana, Slovenia. Tel: +386-1-522-6171. \\ Fax: +386-1-540-1137. E-mail: mihael.rogac@kclj.si
}

\begin{abstract}
Preterm birth is the leading cause of mortality in newborn infants and can lead to significant neonatal morbidities. Spontaneous preterm birth accounts for at least $50.0 \%$ of all preterm births. We argue that chronic maternal stress load, which is an important risk factor for spontaneous preterm birth, could be represented by epigenetic signature of several specific genetic loci in the mother's blood. A literature search was done in PubMed with the following keywords: "DNA methylation," "epigenetics," "maternal stress" and "preterm birth" from year 2000 to 2017 . We suggest that these genetic loci might be related to vulnerability and hypersensibility of stress response during pregnancy in women with preterm births. The mother's epi-genetic stress bioprofile was supposed to be a result of chronic maternal stress load since her birth. This epigenetic bioprofile might also be a potential biomarker for spontaneous preterm birth. DNA methylation changes are tissue-specific and human stress response manifests mostly through the central nervous system (CNS). Nevertheless, we found evidence that methylation changes of DNA isolated from blood leucocytes might be a reliable measure of stress-related epigenetic changes that occur in the CNS. Evaluating biological mechanisms through the development of simple assays based on epigenetic changes to measure chronic stress loads in expectant mothers can lead to our ability to prepare more effective measures for the prevention of preterm births, as well as leading to more effective treatment strategies for both expectant mothers and their newborns.
\end{abstract}

Keywords: Epigenetics; Hypothalamic-pituitaryadrenal (HPA) axis; Maternal stress; Preterm birth.

$\overline{\text { Clinical Institute of Medical Genetics, University Medical Center }}$ Ljubljana, Ljubljana, Slovenia

\section{INTRODUCTION}

The rate of preterm birth, defined as birth before 37 weeks' gestation, is rising worldwide. It accounts for $75.0 \%$ of perinatal mortality and more than half the longterm morbidity. The frequency and severity of adverse outcomes are rising with decreasing gestational age and decreasing quality of care. The preterm birth rate has also risen in most industrialized countries, despite increasing knowledge of risk factors and mechanisms related to preterm birth, and the introduction of many public health and medical interventions designed to reduce preterm birth [1]. The frequency of preterm birth is about $12.0-13.0 \%$ in the USA and 5.0-9.0\% in many other developed countries [2].

Spontaneous preterm birth accounts for at least $50.0 \%$ of all preterm birth. A previous spontaneous preterm birth is the greatest risk factor for spontaneous preterm birth [2]. Chronic maternal stress is increasingly recognized as one of the contributing risk factors for spontaneous preterm birth [3-5]. Thus, preterm birth and chronic maternal stress load during pregnancy are closely connected. We have not, as yet, found the biological mechanisms of stress that relate to the triggering of preterm births, but we can identify several risk factors and behaviors that are connected to spontaneous preterm birth and chronic maternal stress. For example, these are previous trauma in childhood, anxiety and depression, experiences with previous labor, low socioeconomic status, low education, and nutrition.

Measures of stressful life events, the perception of stress, depressive symptoms, and levels of pregnancy-related anxiety are commonly used to indicate maternal adversity. Chronic maternal stress load has been a sum of adverse mother's life events since her birth. Epigenetic biomarkers of several specific genetic loci could be a reliable measure of this chronic maternal stress load. These epigenetic biomarkers may reveal mother's stress bioprofile or stress 
diathesis. Studies showed that there is no strong scientific proof for either the role of abnormal response of corticotropin-releasing hormone (CRH), cytokines or for the role of catecholamines in the pathogenesis of preterm birth [6,7]. Epigenetic modifications interacting with genetic variation to precipitate disease [8] can provide a hypothetical explanation for stress-related disorders such as preterm birth.

DNA methylation changes are tissue-specific. Evidence show that blood/leucocytes might be a possible surrogate through which to investigate stress related conditions that act through the central nervous system (CNS). We suggest that methylation changes of DNA isolated from blood leucocytes are a reliable enough measure of stress related changes that occur in the brain.

Understanding the molecular physiology of chronic maternal stress load in preterm birth has important implications for the development of preventive and treatment measures for preterm birth and for decreasing mortality and morbidity in preterm newborns. Additionally, such understanding could also enable us to develop simple assays based on epigenetic changes, thus providing us with a process that also enables the measurement of chronic stress load in expectant mothers.

Chronic Maternal Stress Load and Preterm Birth. Almost 60 years ago, previous research already revealed that women who were considered as emotionally well-adjusted had a relatively low incidence of difficult labor [9]. Wortis and Freedman [10] found that the endemic nature of premature delivery in women of low social class was similarly related to the stress of life experiences. Women who react with greater sensitivity and less resilience to their life situations also appear more likely to deliver prematurely [10]. Chronic or recurrent stress that occurs with maternal under nutrition, immune system response, early-life events or maternal psychopathology, leads to cascading, potentially irreversible changes in biological stress-regulatory systems
[11]. Accumulated stress appears to negatively impact our ability to respond to stress, and also affects how we perceive stress in relation to our emotional response to environment. Adaptation to stress has its own consequences, including outcomes such as preterm birth.

Early life experiences appear to increase human susceptibility for anxiety and depression that are known risk factors for preterm birth [12-14]. Chronic stressors are recognized for being particularly salient among poor and minority women, that is, women who also correspondingly experience the highest rates of adverse birth outcomes. Expectant mothers from lower socio-economic groups are often exposed to a higher incidence of incomplete families, poor housing, low educational level, high mobility, dysfunctional families and social pathology [10]. We know that for individual subjects within this group, such factors often represent an accumulation of psychological stresses. Evaluating relationship between chronic maternal stress load and spontaneous preterm birth, Manuck et al. [15] identified nine potential spontaneous preterm birth clinical phenotypes based on clinical data. Evidence of any type of maternal stress was associated with $59.8 \%$ in a group of very early spontaneous preterm birth and with $55.4 \%$ in a group of early spontaneous preterm birth.

Chronic Maternal Stress Load and Epigenetics. We now explain how DNA methylation is involved in regulating the human organism's stress response. The hypothalamic-pituitary-adrenal (HPA) axis is a major component of human stress response and its vulnerability is a key factor in the pathogenesis of many chronic diseases of adulthood. The HPA axis vulnerability is related to the velocity, amplitude and duration of mother's stress response. We suggest describing this as HPA axis hyper-sensibility, as stress response is often quick, strong and too long in mothers with chronic stress loads (Figure 1). Most commonly studied genes related to stress response are $\mathrm{NR} 3 \mathrm{Cl}$

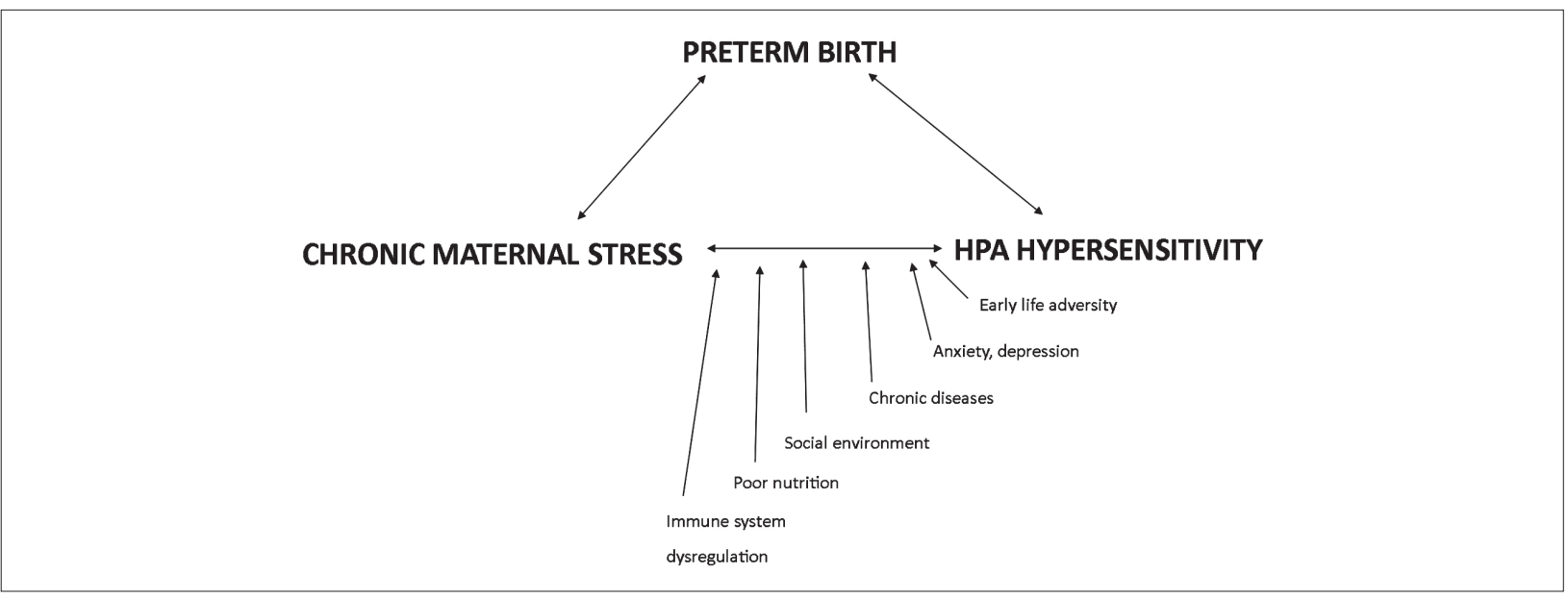

Figure 1. Influence of specific risk factors on chronic maternal stress that has effect on HPA hypersensitivity and can lead to preterm birth. 
which encodes the glucocorticoid receptor; 11B-HSD2 which encodes an enzyme to regulate the transmission of cortisol to the fetus at the maternal-fetal boundary; FKBP5 which encodes $F K 506$, a negative regulator of cortisol response; $B D N F$, which influences neuronal development; and, serotonin transporter SLC6A4 which encodes 5-HTT protein, an important regulator of emotional behavior responses in early life experiences.

Existing studies have primarily investigated the role of glucocorticoid receptor expression and sensibility, which is related to the promoter $N R 3 C 1$ methylation changes [16-20]. DNA methylation of few other genes such as FKBP5, BDNF, $11 B-H S D 2$, and serotonin transporter SLC6A4 could also be involved. Perhaps these stressrelated molecules also influence the activity of HPA axis and/or human organism's response to stress [21-23]. For example, a deficiency of $11 B-H S D 2$ leads to overexposure of the fetus to cortisol and lower birth weight [24]. Placental $11 B H S D 2$ is dynamically regulated by proinflammatory cytokines, malnutrition, and maternal stress or anxiety [25]. We suggest that DNA methylation changes in these genes occur after increased exposure to cortisol, especially in fetal and infant periods of life. Why and how these changes occur is still a topic of discussion.

In animals, however, maternal licking/grooming (LG) behavior has effect on offspring stress responses by increasing HPA axis responses to stress. This kind of mother's behavior increases glucocorticoid receptor expression and negative feedback. These changes in the offspring result from epigenetic alterations, including DNA demethylation and increased histone acetylation [2]. Subsequent studies in humans expanded on the findings in rats. Turecki and Meaney [27] systematically reviewed the effects of the social environment and stress on GR gene methylation. They found that nine out of 10 human studies examining exon $1_{\mathrm{F}}$ methylation $\left(1_{7}\right.$ homologue in rats) reported increased promoter methylation with early life adversity. Early life adversity is a known cause of anxiety and depression in pregnancy. Glucocorticoid receptor DNA methylation is an important step in organism susceptibility to stress and is involved in interaction between early life stressors and higher stress reactivity.

Nevertheless, these processes are related to the placenta physiology and to the role of $11 B H S D 2$ gene methylation, that is the anatomical and physiological connection between mother and fetus. This connection may either protect or be detrimental to the fetus or embryo. DNA methylation changes in other genes such as FKBP5, BDNF and serotonin transporter SLC6A4, could also be a part of mother's stress bioprofile and could have a role in explaining biological mechanisms of preterm birth.

Paquette et al. [23,28] studied in 509 infants the influence of placental FKBP5 epigenetic variation at intron 7, which is associated with infant neurobehavioral outcome in the neonatal intensive care unit (NICU). FKBP5 also regulates cortisol response within the placenta. Infants who were born with an elevated expression of FKBP5 showed elevated stress abstinence at the time of birth. DNA methylation changes of the $B D N F$ gene were studied in cases of early-life adversity and in psychiatric conditions [29-31]. Montirosso et al. [32] and Provenzi et al. [22] evaluated serotonin transporter SLC6A4 in pain-related stress during NICU stays and its effect on infants' temperament at 3 months of age. Preterm birth per se was not found to be associated with epigenetic alterations of the SLC6A4, whereas higher levels of pain-related stress exposure in NICU stays may alter transcriptional activity of the serotonin transporter gene. Hillman et al. [33] investigated the feto-placental genome and its interaction with the maternal in utero environment leading to poor placental development and fetal growth restriction. They found that growth-restricted neonates have distinct DNA methylation profiles in preterm placenta and in cord blood at birth. Sparrow et al. [34] revealed that preterm birth is associated with alterations in the methylome at sites that influence neural development and function.

Epigenetic changes of several specific genetic loci may comprise parts of the larger metabolic network, where hypersensibility of the HPA axis is just one side of the story (Figure 2). Hypersensibility of the HPA axis is not necessarily manifested by increased cortisol concentration in the blood, but may be related to receptor changes in the hormones and neurotransmitters related to human stress response. Preterm birth may, therefore, be identifiable as a clinical symptom of chronic maternal stress load that has

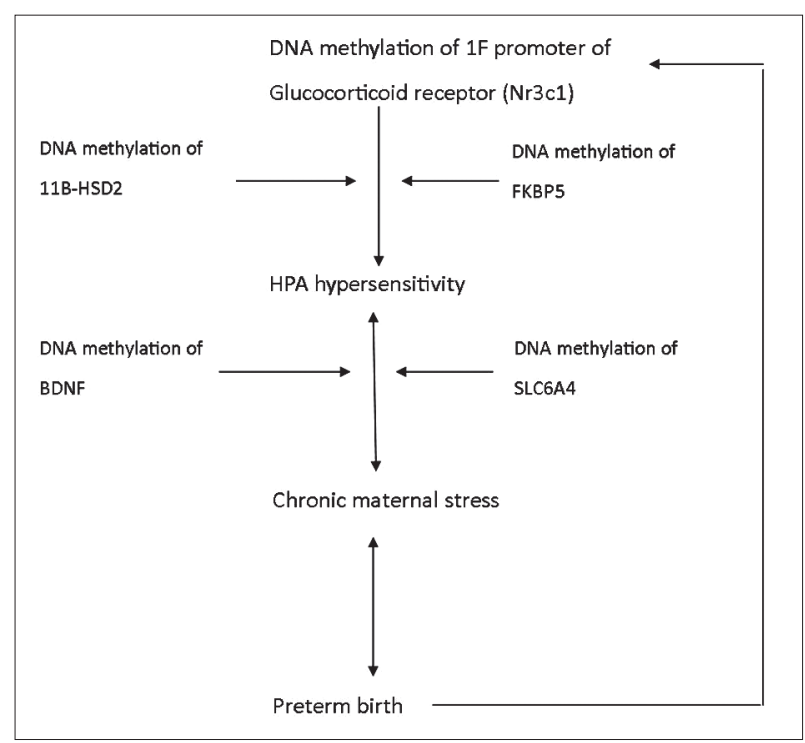

Figure 2. Suggested epigenetic mechanisms that can lead to HPA hypersensitivity and preterm birth. 
accumulated since mother's birth and is part of mother's stress bioprofile. Studies involving preterm birth and DNA methylation changes in specific genetic loci can, therefore, provide us with new opportunities and research challenges.

Epigenetics and Preterm Birth. Several studies have examined the associations between the epigenome and preterm birth. In support for this hypothesis, Vidal et al. [8] found that maternal stress may be associated with epigenetic changes at MEST DMR, a gene relevant to maternal care and obesity. Reduced prenatal stress may support the epigenomic profile of a healthy infant [8].

Three studies have investigated $\mathrm{CpG}$ sites connected to preterm birth using neonatal blood [35-37]. Parets et al. [35] found $29 \mathrm{CpG}$ sites associated with development and the Notch signaling pathway that were associated to preterm birth in a case control study of 50 African American neonates. These sites were independent of gestational age [35]. Cruickshank et al. [36] also investigated blood of premature neonates, and they identified $1555 \mathrm{CpG}$ sites associated with preterm birth; no specific type of preterm birth was defined. These differences were no longer detectable at age 18 [36]. Fernando et al. [37] identified 1855 sites associated with spontaneous preterm birth from cord blood, and 196 were independent of gestational age and none of these overlapped with the 29 preterm birth associated sites from the previous study of Parets et al. [35].

There have been few studies examining DNA methylation differences in mothers who deliver preterm. Parets et al. [38] found that neonatal methylation could be predicted from maternal methylation and these $\mathrm{CpG}$ sites were enriched in biological pathways implicated in preterm birth and chronic diseases. Heng et al. [39] examined 469 genes that were differentially expressed in 106 women delivering preterm compared to 48 women with threatened preterm birth. These genes were correlated with several pathways, including stress response and mRNA processing [39].

Many other studies examined other types of biomarkers for preterm birth such as cytokines and other metabolites in maternal serum, but biomarkers that examine DNA methylation changes may allow for earlier identification of those at increased risk for preterm birth. DNA methylation changes are also more suitable to screening with next-generation sequencing panels that utilize standardized chemistry, and are able for more rapid and reproducible assessment of multiple biomarkers. However, evidence of preterm birth's epigenetic signature is still scarce and studies are so far inconclusive.

Epigenetic Signature in Blood as a Biomarker for Preterm Birth. A limitation of studies that evaluate epigenetic biomarkers for stress related clinical conditions is that DNA methylation changes were investigated in the blood and not in the brain. It is certainly not necessary that a potentially useful biomarker detected in peripheral blood resembles expression of the same analyte in the brain. To date, most relevant studies have focused specifically on the correspondence of the "methylome" (all methylated sites) across tissues.

Measuring DNA methylation changes in single genes, as in promoter I and IV of BDNF and FKBP 5 genes, and comparing blood and brain tissue, indicate that blood is a good proxy for brain in terms of cytosine methylation measured at the genome wide level $[40,41]$. For example, in schizophrenia patients, Murphy et al. [42] found nearly identical patterns of $\mathrm{CpG}$ site-specific methylation across the blood and brain samples analyzing promoter region of a single catechol-O-methyltransferase (COMT) gene. These data adequately indicate the correlation between DNA methylation in peripheral blood leukocytes and CNS samples.

Researchers mostly studied the whole methylome across various tissues. Fan and Zhang [43] reported strong positive correlations in $\mathrm{CpG}$-island methylation status across all somatic tissues but they did not included brain tissue in the study. Davies et al. [44] observed that $\mathrm{CpG}$ sites within islands were more correlated across the cortex, the cerebellum and blood than sites within island shores or coding sequencing. Van der Oord et al. [45] found that when they analyzed DNA extracted from 1408 blood samples and 66 postmortem brain samples of schizophrenia cases and controls, that of the $\mathrm{CpG}$ single nucleotide polymorphism (SNP) methylated in brain, 94.0\% were also methylated in the blood. A review of the epigenomic literature by Tylee et al. [46] revealed that CpG-island methylation levels are generally highly correlated $(r=$ 0.90 ) between blood and brain, and their review of the transcriptome studies suggest that between 35.0 and $80.0 \%$ of known transcripts are present in both brain and blood tissue samples.

Recent studies also suggest that certain blood cells such as lymphocytes and monocytes are a reliable source of DNA methylation changes in the blood. And these changes are correlated with DNA methylation changes in the brain. An increasing body of evidence suggests that there is a close relationship between the CNS and the immune system. Lymphocytes appear to play a central role in this communication. Numerous studies have shown similarities between receptor expression and mechanisms of the transduction processes of cells in the nervous system and lymphocytes. In several neuropsychiatric disorders such as depression, stress, Alzheimer's disease and schizophrenia, researchers found alterations of metabolism and cellular functions in the CNS as well as main neurotransmitter and hormonal systems that are similar to altered function and metabolism of blood lymphocytes [47-49]. The presence 
of sympathetic fibers in lymphoid tissues suggests that direct contact occurs for neural signaling cascades with the immune system [50].

An important limitation in all of these studies was that brain DNA methylation changes were studied in postmortem tissue. Because taking a blood sample is a minimally invasive procedure, it is typically considered as a practical surrogate. For the present, however, such a practice affords a reliable enough procedure for the examination of DNA methylation changes in peripheral blood for evaluating the risk of preterm birth as one kind of various stress-related disorder.

Implications. Our analyses suggest that DNA methylation changes of specific genetic loci cause increased vulnerability and sensitivity of the human stress response. This exaggerated stress response can also manifest in preterm birth. It is possible that part of an exaggerated response to stress in preterm birth lies in the DNA methylation pattern of specific genes involved in cortisol signaling and neurotransmitter systems such as serotonin.

Finding epigenetic biomarkers of increased stress susceptibility for preterm birth or mother's stress bioprofile would be a first step in characterizing that group of women with preterm births. Based on the results of a blood sample, we could begin preventive and therapeutic measures to decrease chronic maternal stress load in this group of women. Preventive measures could involve cognitive behavior therapy support, social assistance for underprivileged groups of women, and mind-body therapies for stress reduction. Therapeutic measures such as drugs that change DNA methylation patterns are also underway. Nutrition, too, might play a role as a preventive measure against chronic stress accumulation.

Investigating chronic maternal stress load and risk for preterm birth is also important in identifying epigenetic mechanisms of human stress response, and fetal programing. Again, in doing so, we may then be able to develop simple assays based on epigenetic changes in order to measure chronic stress load in expectant mothers.

Declaration of Interest. The authors report no conflicts of interest. The authors alone are responsible for the content and writing of this article.

\section{REFERENCES}

1. Goldenberg RL, Rouse DJ. Prevention of premature birth. N Engl J Med. 1998; 339(5): 313-320.

2. Goldenberg RL, Culhane JF, Iams JD, Romero R. Epidemiology and causes of preterm birth. Lancet. 2008; 371(9606): 75-84.
3. Coletti MF, Caravale B, Gasparini C, Franco F, Campi F, Dotta A. One-year neurodevelopmental outcome of very and late preterm infants: Risk factors and correlation with maternal stress. Infant Behav Dev. 2015; 39(5): 11-20.

4. Gennaro S, Hennessy MD. Psychological and physiological stress: Impact on preterm birth. J Obstet Gynecol Neonatal Nurs. 2003; 32(5): 668-675.

5. Lobel M, Cannella DL, Graham JE, DeVincent C, Schneider J, Meyer BA. Pregnancy-specific stress, prenatal health behaviors, and birth outcomes. Health Psychol. 2008; 27(5): 604-615.

6. Harville EW, Savitz DA, Dole N, Herring AH, Thorp JM. Stress questionnaires and stress biomarkers during pregnancy. J Womens Health (Larchmt). 2009; 18(9): 1425-1433.

7. Kramer MS, Lydon J, Goulet L, Kahn S, Dahhou M, Platt RW, et al. Maternal stress/distress, hormonal pathways and spontaneous preterm birth. Paediatr Perinat Epidemiol. 2013; 27(3): 237-246.

8. Vidal AC, Benjamin Neelon SE, Liu Y, Tuli AM, Fuemmeler BF, Hoyo C, et al. Maternal stress, preterm birth, and DNA methylation at imprint regulatory sequences in humans. Genet Epigenet. 2014; 6(9): 37-44.

9. Scott EM, Thomson AM. A psychological investigation of primigravidae. IV. Psychological factors and the clinical phenomena of labour. J Obstet Gynaecol Br Emp. 1956; 63(4): 502-508.

10. Wortis H, Freedman AM. Maternal stress and premature delivery. Bull World Health Organ. 1962; 26 : 285-291.

11. Rubin LP. Maternal and pediatric health and disease: Integrating biopsychosocial models and epigenetics. Pediatr Res. 2016; 79(1-2): 127-135.

12. Staneva A, Bogossian F, Pritchard M, Wittkowski A. The effects of maternal depression, anxiety, and perceived stress during pregnancy on preterm birth: A systematic review. Women Birth. 2015; 28(3): 179-193.

13. Sanchez SE, Puente GC, Atencio G, Qiu C, Yanez D, Gelaye B, et al. Risk of spontaneous preterm birth in relation to maternal depressive, anxiety, and stress symptoms. J Reprod Med. 2013; 58(1-2): 25-33.

14. McDonald SW, Kingston D, Bayrampour H, Dolan SM, Tough SC. Cumulative psychosocial stress, coping resources, and preterm birth. Arch Womens Ment Health. 2014; 17(6): 559-568.

15. Manuck TA, Esplin MS, Biggio J, Bukowski R, Parry $\mathrm{S}$, Zhang $\mathrm{H}$, et al. The phenotype of spontaneous pre- 
term birth: Application of a clinical phenotyping tool. Am J Obstet Gynecol. 2015; 212(4): 487.e1-487.e11.

16. Braithwaite EC, Kundakovic M, Ramchandani PG, Murphy SE, Champagne FA. Maternal prenatal depressive symptoms predict infant NR3C1 $1 \mathrm{~F}$ and BDNF IV DNA methylation. Epigenetics. 2015; 10(5): 408-417.

17. Conradt E, Fei M, LaGasse L, Tronick E, Guerin $\mathrm{D}$, Gorman D, et al. Prenatal predictors of infant self-regulation: The contributions of placental DNA methylation of $\mathrm{NR} 3 \mathrm{C} 1$ and neuroendocrine activity. Front Behav Neurosci. 2015; 9(5): 130-135.

18. Schechter DS, Moser DA, Paoloni-Giacobino A, Stenz L, Gex-Fabry M, Aue T, et al. Methylation of NR3C1 is related to maternal PTSD, parenting stress and maternal medial prefrontal cortical activity in response to child separation among mothers with histories of violence exposure. Front Psychol. 2015; 6(5): 690-702.

19. Mulligan CJ, D’Errico NC, Stees J, Hughes DA. Methylation changes at NR3C1 in newborns associate with maternal prenatal stress exposure and newborn birth weight. Epigenetics. 2012; 7(8): 853-857.

20. Perroud N, Paoloni-Giacobino A, Prada P, Olie E, Salzmann A, Nicastro R, et al. Increased methylation of glucocorticoid receptor gene (NR3C1) in adults with a history of childhood maltreatment: A link with the severity and type of trauma. Transl Psychiatry. 2011; 1(12): e59-e68.

21. Non AL, Binder AM, Barault L, Rancourt RC, Kubzansky LD, Michels KB. DNA methylation of stressrelated genes and LINE-1 repetitive elements across the healthy human placenta. Placenta. 2012; 33(3): 183-187.

22. Provenzi L, Fumagalli M, Sirgiovanni I, Giorda R, Pozzoli U, Morandi F, et al. Pain-related stress during the Neonatal Intensive Care Unit stay and SLC6A4 methylation in very preterm infants. Front Behav Neurosci. 2015; 9(4): 99-108.

23. Paquette AG, Lester BM, Koestler DC, Lesseur C, Armstrong DA, Marsit CJ. Placental FKBP5 genetic and epigenetic variation is associated with infant neurobe-havioral outcomes in the RICHS cohort. PLoS One. 2014; 9(8): e104913.

24. O’Donnell KJ, Meaney MJ. Fetal origins of mental health: The developmental origins of health and disease hypothesis. Am J Psychiatry. 2017; 174(4): 319-328.

25. Chapman K, Holmes M, Seckl J. 11Beta-hydroxysteroid dehydrogenases: Intracellular gate-keepers of tissue glucocorticoid action. Physiol Rev. 2013; 93(3): 1139-1206.

26. Champagne FA, Weaver IC, Diorio J, Dymov S, Szyf M, Meaney MJ. Maternal care associated with methylation of the estrogen receptor-alphalb promoter and estrogen receptor-alpha expression in the medial preoptic area of female offspring. Endocrinology. 2006; 147(6): 2909-2915.

27. Turecki G, Meaney MJ. Effects of the social environment and stress on glucocorticoid receptor gene methylation: A systematic review. Biol Psychiatry. 2016; 79(2): 87-96.

28. Paquette AG, Lester BM, Lesseur C, Armstrong DA, Guerin DJ, Appleton AA, et al. Placental epigenetic patterning of glucocorticoid response genes is associated with infant neurodevelopment. Epigenomics. 2015; 7(5): 767-779.

29. D'Addario C, Dell'Osso B, Palazzo MC, Benatti B, Lietti L, Cattaneo E, et al. Selective DNA methylation of BDNF promoter in bipolar disorder: differences among patients with BDI and BDII. Neuropsychopharmacology. 2012; 37(7): 1647-1655.

30. Fuchikami M, Morinobu S, Segawa M, Okamoto Y, Yamawaki S, Ozaki N, et al. DNA methylation profiles of the brain-derived neurotrophic factor (BDNF) gene as a potent diagnostic biomarker in major depression. PLoS One. 2011; 6(8): e23881.

31. Unternaehrer E, Luers P, Mill J, Dempster E, Meyer $\mathrm{AH}$, Staehli S, et al. Dynamic changes in DNA methylation of stress-associated genes (OXTR, BDNF) after acute psychosocial stress. Transl Psychiatry. 2012; 2(8): e150-e157.

32. Montirosso R, Provenzi L, Fumagalli M, Sirgiovanni I, Giorda R, Pozzoli U, et al. Serotonin transporter gene (SLC6A4) methylation associates with Neonatal Intensive Care Unit stay and 3-month-old temperament in preterm infants. Child Dev. 2016; 87(1): $38-48$.

33. Hillman SL, Finer S, Smart MC, Mathews C, Lowe $\mathrm{R}$, Rakyan VK, et al. Novel DNA methylation profiles associated with key gene regulation and transcription pathways in blood and placenta of growth-restricted neonates. Epigenetics. 2015; 10(1): 50-61.

34. Sparrow S, Manning JR, Cartier J, Anblagan D, Bastin ME, Piyasena $\mathrm{C}$, et al. Epigenomic profiling of preterm infants reveals DNA methylation differences at sites associated with neural function. Transl Psychiatry. 2016; 6(1): e716-e724.

35. Parets SE, Conneely KN, Kilaru V, Fortunato SJ, Syed TA, Saade G, et al. Fetal DNA methylation associates 
with early spontaneous preterm birth and gestational age. PLoS One. 2013; 8(6): e67489.

36. Cruickshank MN, Oshlack A, Theda C, Davis PG, Martino D, Sheehan P, et al. Analysis of epigenetic changes in survivors of preterm birth reveals the effect of gestational age and evidence for a long term legacy. Genome Med. 2013; 5(10): 96-108.

37. Fernando F, Keijser R, Henneman P, van der KevieKersemaekers AM, Mannens MM, van der Post JA, et al. The idiopathic preterm delivery methylation profile in umbilical cord blood DNA. BMC Genomics. 2015; 16(9): 736-748.

38. Parets SE, Conneely KN, Kilaru V, Menon R, Smith AK. DNA methylation provides insight into intergenerational risk for preterm birth in African Americans. Epigenetics. 2015; 10(9): 784-792.

39. Heng YJ, Pennell CE, Chua HN, Perkins JE, Lye SJ. Whole blood gene expression profile associated with spontaneous preterm birth in women with threatened preterm labor. PLoS One. 2014; 9(5): e96901.

40. Ewald ER, Wand GS, Seifuddin F, Yang X, Tamashiro KL, Potash JB, et al. Alterations in DNA meth-ylation of Fkbp5 as a determinant of blood-brain correlation of glucocorticoid exposure. Psychoneuroendocrinology. 2014; 44(6): 112-122.

41. Aberg KA, Xie LY, McClay JL, Nerella S, Vunck S, Snider S, et al. Testing two models describing how methylome-wide studies in blood are informative for psychiatric conditions. Epigenomics. 2013; 5(4): 367-377.

42. Murphy BC, O’Reilly RL, Singh SM. Site-specific cytosine methylation in S-COMT promoter in 31 brain regions with implications for studies involving schizophrenia. Am J Med Genet B Neuropsychiatr Genet. 2005; 133B(1): 37-42.
43. Fan $\mathrm{S}$, Zhang X. CpG island methylation pattern in different human tissues and its correlation with gene expression. Biochem Biophys Res Commun. 2009; 383(4): 421-425.

44. Davies MN, Volta M, Pidsley R, Lunnon K, Dixit A, Lovestone $\mathrm{S}$, et al. Functional annotation of the human brain methylome identifies tissue-specific epigenetic variation across brain and blood. Genome Biol. 2012; 13(6): R43-R57.

45. van den Oord EJ, Clark SL, Xie LY, Shabalin AA, Dozmorov MG, Kumar G, et al. A whole methylome CpG-SNP association study of psychosis in blood and brain tissue. Schizophr Bull. 2016; 42(4): 1018-1026.

46. Tylee DS, Kawaguchi DM, Glatt SJ. On the outside, looking in: A review and evaluation of the comparability of blood and brain "-omes". Am J Med Genet B Neuropsychiatr Genet. 2013; 162B(7): 595-603.

47. Irwin M. Immune correlates of depression. Adv Exp Med Biol. 1999; 461: 1-24.

48. Maes M, Bosmans E, Calabrese J, Smith R, Meltzer HY. Interleukin-2 and interleukin-6 in schizophrenia and mania: Effects of neuroleptics and mood stabilizers. J Psychiatr Res. 1995; 29(2): 141-152.

49. Schwarz MJ, Müller N, Riedel M, Ackenheil M. The Th2-hypothesis of schizophrenia: A strategy to identify a subgroup of schizophrenia caused by immune mechanisms. Med Hypotheses. 2001; 56(4): 483-486.

50. Felten DL, Felten SY, Bellinger DL, Carlson SL, Ackerman KD, Madden KS, et al. Noradrenergic sympathetic neural interactions with the immune system: Structure and function. Immunol Rev. 1987; 100(12): 225-260. 